\title{
Lidocaine inhibits the proliferation and metastasis of epithelial ovarian cancer through the Wnt/ $\beta$-catenin pathway
}

\author{
Mei Sun ${ }^{1 \#}$, Saisai Huang ${ }^{2 \#}$, Yongtao Gao ${ }^{2}$ \\ ${ }^{1}$ Department of Anesthesiology, the Affiliated Nanjing Jiangbei Hospital of Nantong University, Nanjing, China; ${ }^{2}$ Department of Anesthesiology, the \\ Affiliated Hospital of Nantong University, Nantong, China \\ Contributions: (I) Conception and design: All authors; (II) Administrative support: All authors; (III) Provision of study materials or patients: All \\ authors; (IV) Collection and assembly of data: All authors; (V) Data analysis and interpretation: All authors; (VI) Manuscript writing: All authors; (VII) \\ Final approval of manuscript: All authors. \\ "These authors contributed equally to this work. \\ Correspondence to: Yongtao Gao. Department of Anesthesiology, the Affiliated Hospital of Nantong University, 20 Xisi Road, Nantong, China. \\ Email: gyt19700114@sina.com.
}

Background: Lidocaine, an amide local anesthetic, has recently been found to have anticancer action in various cancer cells. However, the role of lidocaine in epithelial ovarian cancer (EOC) remains largely unknown. In the present study, we investigated how lidocaine regulates the progression of EOC.

Methods: Real-time polymerase chain reaction was used to examine the expression of Snail, Wnt, $\beta$-catenin, E-cadherin, vimentin, matrix metalloproteinase (MMP)-7, MMP-9, and vascular endothelial growth factor in lidocaine-treated cells. Cell proliferation assays, cell apoptosis assays, and cell migration assays were employed to verify the function of lidocaine in EOC cells. Cell proliferation and cell migration assays were employed to verify the function of $W n t / \beta$-catenin signaling in lidocaine-treated EOC cells together with Wnt-overexpressing plasmids or inhibitor NVP-XAV939.

Results: Lidocaine could inhibit proliferation, migration, and invasion, and induce apoptosis in ovarian cancer cells lines in a dose-dependent manner. Wnt/ $\beta$-catenin signaling was involved in the suppression of epithelial-mesenchymal transition progression of ovarian cancer cells, which resulted in the downregulation of Snail and vimentin, as well as the upregulation of E-cadherin. Furthermore, overexpressed Wnt could reverse the carcinostatic effect of lidocaine, while Wnt inhibitor XAV-939 synergistically enhanced the antitumor effect of lidocaine.

Conclusions: Mechanistically, lidocaine could inhibit the proliferation and metastasis of EOC by the Wnt/ $\beta$-catenin pathway to regulate the progression of EOC.

Keywords: Lidocaine; epithelial ovarian cancer (EOC); Wnt/ß-catenin; metastasis

Submitted Apr 27, 2021. Accepted for publication Jul 14, 2021.

doi: $10.21037 /$ tcr-21-1047

View this article at: https://dx.doi.org/10.21037/tcr-21-1047

\section{Introduction}

Epithelial ovarian cancer (EOC) is the seventh most common cancer and eighth most common cause of cancer death among women $(1,2)$. Approximately 230,000 women worldwide are diagnosed and 150,000 die from EOC annually (3). Some deleterious mutations in breast cancer type (BRCA)1 and BRCA2 are largely associated with high-grade serous EOC subtype susceptibility (4). The World Health Organization classification guidelines list 7 EOC histotypes: high-grade serous, low-grade serous, endometrioid, mucinous, clear cell, carcinosarcoma, and malignant Brenner tumors (5). Considerable efforts have been made to implement early screening and detection of EOC in patients; however, EOC oncogenesis in different 
pathological patterns is distinct. Understanding the detailed molecular mechanism is imperative for developing more effective strategies for EOC diagnosis and treatment.

Lidocaine, a derivative of cocaine, is an amide local anesthetic (6). It has a biphasic effect of excitation and inhibition on the central nervous system, and there is no precursory excitation. It is widely used in regional anesthesia, peripheral nerve blocks, and epidural anesthesia with excellent effects on pain management. Lidocaine also has many other pharmacological effects, such as strong anti-inflammatory effects, and can inhibit pyroptosis, protect neurons, and prevent mitochondrial dysfunction (7-10). In a recently published study, lidocaine was found to have antitumor properties in diverse cancers, including hepatocellular carcinoma, malignant glioma, transient receptor potential cation channel subfamily $\mathrm{V}$ member 6 (TRPV6)-expressing cancer, colon adenocarcinoma, bladder cancer, gastric cancer, breast cancer, and lung cancer (6,11-17). Lidocaine can alleviate cytotoxicity resistance in lung cancer cells via the downregulation of microRNA-21, and also significantly inhibit the proliferation of lung cancer by regulating the expression of Golgi transport $1 \mathrm{~A}$ (GOLT1A) $(17,18)$ Another study found that lidocaine can also inhibit the proliferation and metastasis of lung cancer cell via the regulation of the miR-539/epidermal growth factor receptor (EGFR) axis (16). In a recent study, lidocaine could inhibit the metastatic potential of EOC cell via inhibiting EMT and FAK/Paxillin pathway, which indicating the potential of lidocaine in treat EOC (19). However, whether there were other potential mechanisms remains to be clarified.

$\beta$-Catenin is a crucial signaling transducer in Wnt signaling. Mutations of canonical Wnt signaling pathway genes frequently occur in cancer and lead to abnormal accumulation of the key effector $\beta$-catenin (20). Wnt/ $\beta$-catenin signaling mainly affects the maintenance of cancer stem cells, metastasis, and immune control (21). Small molecular inhibitors of the $\mathrm{Wnt} / \beta$-catenin pathway have been shown to inhibit Wnt secretion, leading to a size reduction of mouse mammary tumor virus (MMTV)-Wnt1-driven tumors and head and neck cancer xenotransplants (22). Therefore, Wnt signaling is strongly associated with cancer.

In the present study, we found that lidocaine could inhibit proliferation, migration, and invasion, and induce apoptosis in ovarian cancer cells lines in a dose-dependent manner. Further investigations demonstrated that lidocaine also could inhibit epithelial-mesenchymal transition (EMT) of ovarian cancer cells via regulation of $\mathrm{Wnt} / \beta$-catenin pathway signaling and EMT-related genes. Furthermore, Wnt overexpression reverses the carcinostatic effect of lidocaine, while Wnt inhibitor XAV-939 enhances it. This suggests that lidocaine could inhibit the proliferation and metastasis of EOC through the $\mathrm{Wnt} / \beta$-catenin pathway.

We present the following article in accordance with the MDAR reporting checklist (available at https://dx.doi. org/10.21037/tcr-21-1047).

\section{Methods}

\section{Cell culture}

Human ovarian cancer cell line, SKOV3 and COC1 were purchased from the American Type Culture Collection (ATCC, Manassas, VA, USA) and cultured in McCoy's $5 \mathrm{~A}$ medium supplemented with $10 \%$ fetal bovine serum, America) for transient transfection. Samples were collected after $24 \mathrm{~h}$ to quantify the mRNA expression of the target gene.

\section{Real-time polymerase chain reaction (PCR)}

TRIzol and microRNA isolation kit were purchased from Qiagen (Valencia, CA, USA). Samples were homogenized in TRIzol, and total RNA extract and reverse transcription were performed according to the manufacturer's protocol. For PCR amplification of the cDNA fragment coding target genes, sense and antisense primer sequences for matrix metalloproteinase (MMP)-7, MMP-9, vascular endothelial growth factor (VEGF), Wnt, $\beta$-catenin, E-cadherin, vimentin, and $\beta$-actin were as described previously (Table 1).

\section{Apoptosis analysis by fuorescence-activated cell sorting}

The Annexin V-fluorescein isothiocyanate (FITC)/ propidium iodide (PI) detection kit (TransGene Biotek, Winooski, VT, USA) was used for the apoptosis assays. The cells were treated and then labelled with Annexin V-FITC and PI at room temperature under dark conditions for $20 \mathrm{~min}$. The apoptosis percentage was tested using a FACSCalibur flow cytometer (Becton Dickinson, San Jose, CA, USA).

\section{Cell counting kit-8 (CCK-8) assay}

SKOV3 and COC1 cells were seeded in a 96-well plate $(3,000 /$ well). Cell proliferation was examined by CCK-8, 
Table 1 The primers in this study

\begin{tabular}{lll}
\hline Primer sequence & Sense & Anti-sense \\
\hline MMP-7 & TCGGAGGAGATGCTCACTTCGA & GGATCAGAGGAATGTCCCATACC \\
MMP-9 & GCCACTACTGTGCCTTTGAGTC & CCCTCAGAGAATCGCCAGTACT \\
VEGF & TTGCCTTGCTGCTCTACCTCCA & GATGGCAGTAGCTGCGCTGATA \\
Wnt & ATGAACCGCCACAACAACGAGG & GTCCTTGAGGAAGTCACCGATG \\
$\beta$-catenin & CACAAGCAGAGTGCTGAAGGTG & GATTCCTGAGAGTCCAAAGACAG \\
E-cadherin & GCCTCCTGAAAAGAGAGTGGAAG & TGGCAGTGTCTCTCCAAATCCG \\
vimentin & AGGCAAAGCAGGAGTCCACTGA & ATCTGGCGTTCCAGGGACTCAT \\
$\beta$-actin & CACCATTGGCAATGAGCGGTTC & AGGTCTTGCGGATGTCCACGT \\
\hline
\end{tabular}

as per the kit's instructions. Absorbance of $450 \mathrm{~nm}$ at $48 \mathrm{~h}$ after seeding was measured by a microplate reader (Bio-Rad, Hercules, CA, USA).

\section{Terminal deoxynucleotidyl transferase-mediated} digoxigenin-dUTP nick-end labeling (TUNEL) assays

TUNEL assay kit was purchased from Abcam (ab66108, Cambridge, USA). Treated cells and fixed cells in 4\% PFA, next $0.1 \%$ Triton $\mathrm{X}-100$ was used to permeabilize cells for $2 \mathrm{~min}$. Cells were incubated with TUNEL reaction mixture under dark conditions for $1 \mathrm{~h}$, and then incubated with Hoechst 33342 for 15 min. Mounted cells with ProLong Gold antifade overnight and imaged cells by a fluorescence microscope.

\section{Cell migration and invasion assays}

SKOV3 and COC1 cells were transfected with Wnt recombinant and blank plasmid, as well as $100 \mu \mathrm{M}$ lidocaine, and $\mathrm{Wnt}$ recombinant plasmid $+100 \mu \mathrm{M}$ lidocaine in 12-well plates (10,000 cells/well) for $24 \mathrm{~h}$. Then 5,000 cells per well were seeded in the inserts pre-equilibrated of 8 - $\mu \mathrm{m}$ pore Transwell for migration assays (Corning, NY, USA). Another, for invasion assay, the insert was coated with Matrigel before the cells were inoculated. After $24 \mathrm{~h}$ of incubation, the inserts were rinsed, fixed in $2 \%$ paraformaldehyde for $10 \mathrm{~min}$, and then stained with crystal violet (Beyotime, Jiangsu, China). Migrated cells were counted under a microscope (ECLIPSE Ti; Nikon,Japan).

\section{Statistical analysis}

Each experiment was performed 3 times, and data were analyzed with GraphPad Prism 8.0 software by 2 -tailed test, unpaired Student's $t$-test or one-way analysis of variance. Differences with $\mathrm{P}<0.05$ were considered statistically significant.

\section{Results}

Lidocaine inbibits proliferation and induces apoptosis in ovarian cancer cells

To examine the effect of lidocaine in ovarian cancer, we selected the SKOV3 and COC1 cell lines and treated the cells with different doses of lidocaine $(1,10$, and $100 \mu M)$ and found that lidocaine inhibits the proliferation of SKOV3 and COC1 cells in a dose-dependent manner (Figure 1A). Furthermore, we performed BrdU (5-Bromodeoxyuridine) test to confirm the effect of lidocaine, and also found that the proliferation of SKOV3 and COC1 cells was inhibited in a dose-dependent manner (Figure 1B). In addition, the apoptosis analysis of SKOV3 cells with 1, 10, and $100 \mu \mathrm{M}$ lidocaine revealed that lidocaine could promote apoptosis in a dose-dependent manner (Figure 1C). These data indicated that lidocaine can suppress the progression of ovarian cancer cells.

\section{Lidocaine inbibits the migration and invasion of ovarian cancer cells lines SKOV3 and COC1}

To determine the effect of lidocaine in the migration and invasion of ovarian cancer cells, we treated SKOV3 and COC1 cells with different doses of lidocaine to analyze migration by Transwell assay. As shown in Figure 2A, the migration rate in SKOV3 or COC1 cells with $1 \mu \mathrm{M}$ lidocaine began to decline compared with that of the 

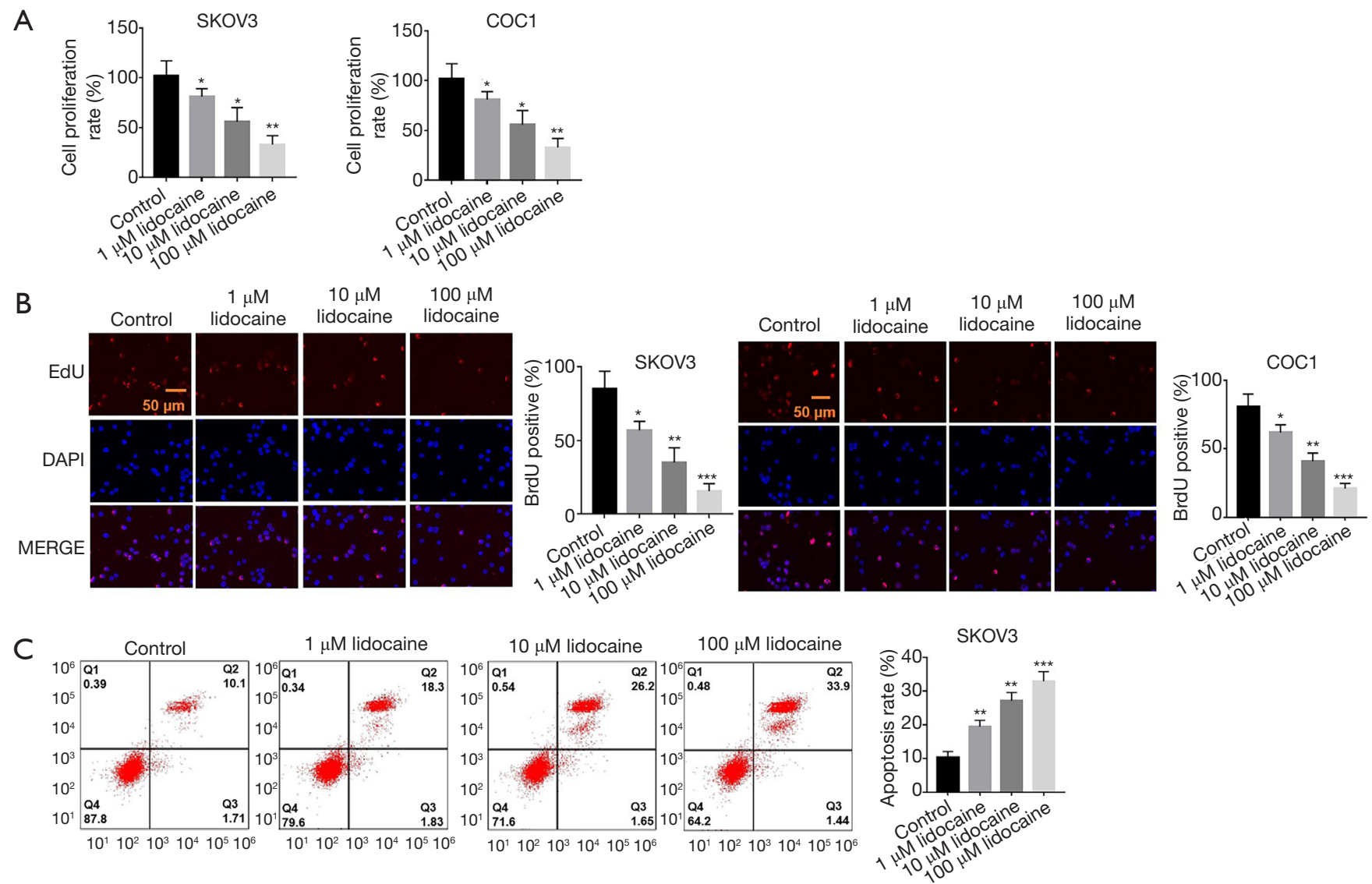

Figure 1 Lidocaine inhibits the proliferation and promote apoptosis of ovarian cancer cell lines. (A) Lidocaine inhibits the proliferation of ovarian cancer cell lines in a dose-dependent manner. (B) Cell proliferation assay for ovarian cancer cells with different doses of lidocaine by BrdU test (scale bar $=50 \mu \mathrm{m})$. (C) Lidocaine promotes apoptosis of ovarian cancer cell line in a dose-dependent manner. ${ }^{*} \mathrm{P}<0.05$, ${ }^{* *} \mathrm{P}<0.01$, ${ }^{* * *} \mathrm{P}<0.001$ : other groups compared with control group.

control group. The migration rate was reduced by 2 -fold in the $10 \mu \mathrm{M}$ lidocaine group, while it was reduced by 4 -fold in the $100 \mu \mathrm{M}$ lidocaine group compared with the control group. We then carried out a Transwell assay to examine cell invasion with different concentrations of lidocaine. These findings demonstrated that lidocaine inhibits the invasion of ovarian cancer cells lines SKOV3 and COC1 in a dose-dependent manner (Figure 2B). As MMP-7, MMP9 , and VEGF were key determinants for cell migration and invasion, we detected their changes in mRNA expression levels by quantitative reverse transcription-PCR (qRTPCR). The findings revealed that the expression levels of MMP-7, MMP-9, and VEGF were also inhibited in ovarian cancer cells lines SKOV3 and COC1 in a dose-dependent manner (Figure 2C,D,E). These data indicated that lidocaine can suppress the migration and invasion of ovarian cancer cells via inhibiting the expression of MMP-7, MMP-9, and VEGF.

\section{Lidocaine inbibits EMT in ovarian cancer cells}

EMT has been shown to be a critical process for the migration, invasion, and metastasis of cancer cells. Therefore, in the present study, we treated ovarian cancer cells with different concentrations of lidocaine to investigate the changes of EMT-associated genes of ovarian cancer cells lines SKOV3 and COC1. Snail, an important transcription factor to determine EMT, was inhibited by lidocaine, and the inhibiting effect was more significant as the lidocaine concentration increased (Figure 3A). Because Wnt and $\beta$-catenin is involved in the EMT process, qRTPCR analysis revealed that the expression level of Wnt and 
A

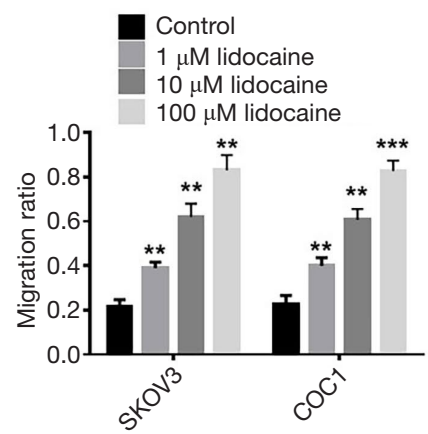

B
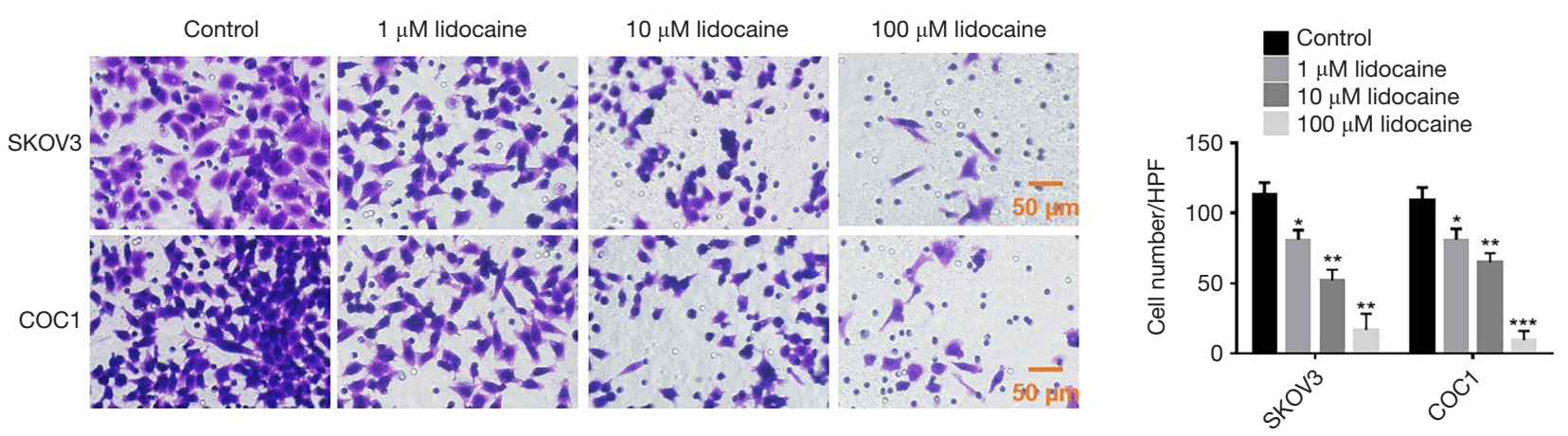

C

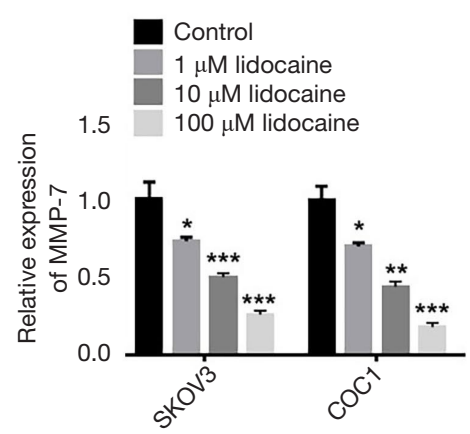

E

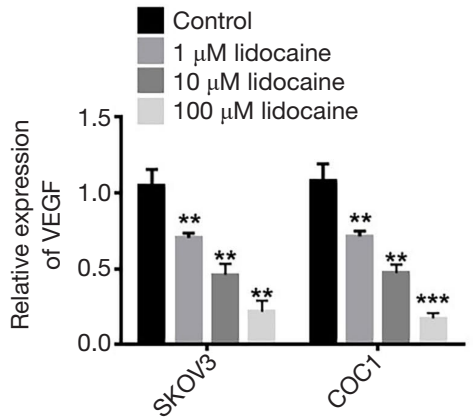

D

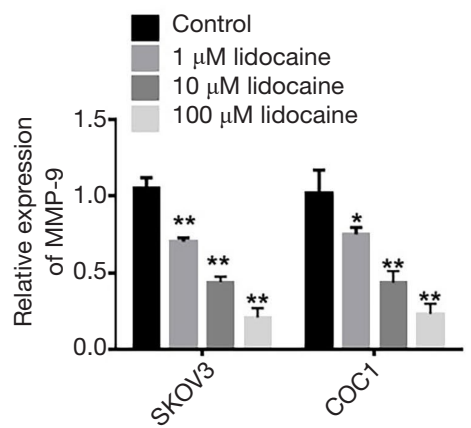

Figure 2 Lidocaine inhibits the migration of ovarian cancer cells lines. (A) Lidocaine inhibits the migration of ovarian cancer cells lines SKOV3 and COC1 in a dose-dependent manner. (B) Lidocaine inhibits the invasion of ovarian cancer cells lines SKOV3 and COC1 in a dose-dependent manner. (C) Quantitative reverse transcription polymerase chain reaction (qRT-PCR) was used to analyze matrix metalloproteinase (MMP)-7 expression of ovarian cancer cells lines SKOV3 and COC1 with different doses of lidocaine. (D) qRT-PCR was used to analyze MMP-7 expression of ovarian cancer cells lines SKOV3 and COC1 with different doses of lidocaine. (E) qRT-PCR was used to analyze vascular endothelial growth factor (VEGF) expression of ovarian cancer cells lines SKOV3 and COC1 with different doses of lidocaine. ${ }^{*} \mathrm{P}<0.05,{ }^{* *} \mathrm{P}<0.01,{ }^{* * *} \mathrm{P}<0.001$ : other groups compared with control group. 
A

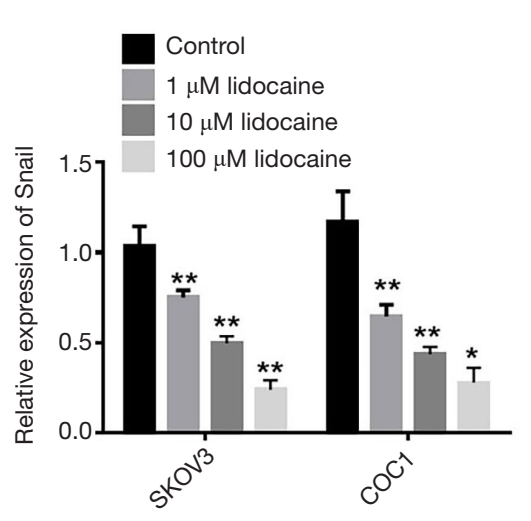

C

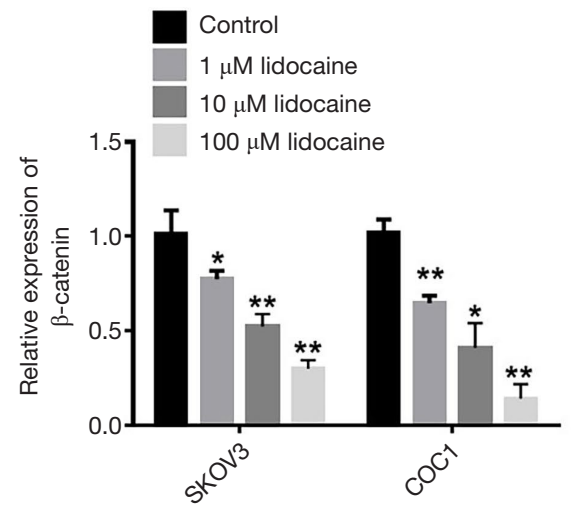

$E$

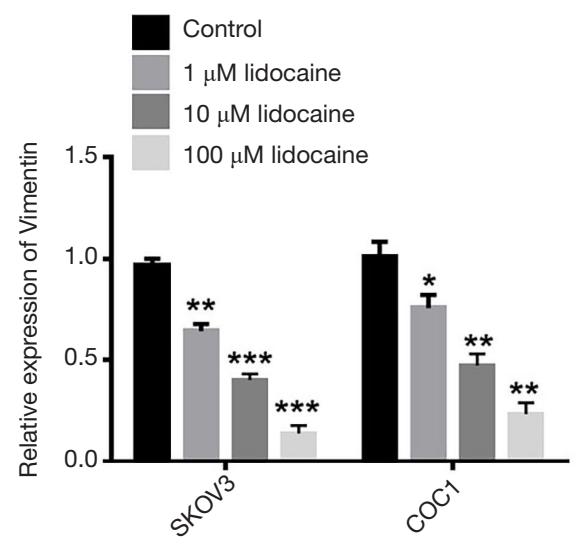

B

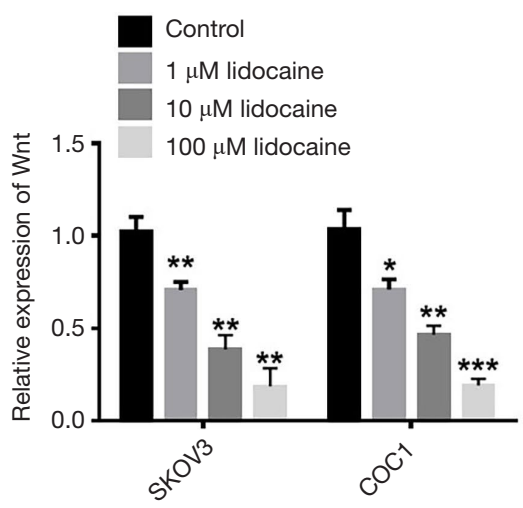

Figure 3 Lidocaine inhibits EMT in ovarian cancer cells. (A) Quantitative reverse transcription polymerase chain reaction (qRT-PCR) was used to analyze the expression of Snail in ovarian cancer cells lines SKOV3 and COC1 with different doses of lidocaine. (B) qRT-PCR was used to analyze the expression of Wnt in ovarian cancer cells lines SKOV3 and COC1 with different doses of lidocaine. (C) qRT-PCR was used to analyze the expression of $\beta$-catenin in ovarian cancer cells lines SKOV3 and COC1 with different doses of lidocaine. (D) Lidocaine enhanced the expression of E-cadherin in ovarian cancer cells lines SKOV3 and COC1 in a dose-dependent manner. (E) Lidocaine inhibited the expression of vimentin in ovarian cancer cells lines SKOV3 and COC1 in a dose-dependent manner. ${ }^{*} \mathrm{P}<0.05$, ${ }^{*} \mathrm{P}<0.01,{ }^{* * *} \mathrm{P}<0.001$ : other groups compared with control group. 
-catenin mRNA was repressed in ovarian cancer cells lines SKOV3 and COC1 with different doses of lidocaine (Figure $3 B, C$ ). In addition, E-cadherin and vimentin, markers of the EMT process, were more significantly inhibited and increased, respectively, as the lidocaine concentration increased compared with the control group (Figure 3D,E). These data indicated that lidocaine dose dependently inhibits EMT progression in ovarian cancer cells.

\section{Overexpression of Wnt reverses the carcinostatic effect of lidocaine}

To further investigate the function of the $\mathrm{Wnt} / \beta$-catenin pathway in the carcinostatic effect of lidocaine, we constructed the Wnt overexpression plasmid and treated SKOV3 cells with blank plasmid, Wnt recombinant plasmid, $100 \mu \mathrm{M}$ lidocaine, and Wnt recombinant plasmid $+100 \mu \mathrm{M}$ lidocaine. Cell scratch test results found that overexpressed Wnt could significantly enhance SKOV3 cell migration ability, while the lidocaine-treated group remarkably suppressed migration ability compared with the control group. The migration ability was obviously reversed in Wnt recombinant plasmid + $100 \mu \mathrm{M}$ lidocainetreated group compared with that in the $100 \mu \mathrm{M}$ lidocainetreated group (Figure 4A). Similarly, Transwell assay results showed that overexpressed Wnt substantially promoted cell invasion capacity, while the lidocaine-treated group considerably inhibited cell invasion capacity compared with the control group. Cell invasion capacity was significantly reversed in Wnt recombinant plasmid + $100 \mu \mathrm{M}$ lidocainetreated group compared with that in the $100 \mu M$ lidocainetreated group (Figure 4B). We also examined the expression level of $W n t$ and $\beta$-catenin mRNA and found that $W n t$ recombinant plasmid reversed the inhibition of lidocaine for Wnt and $\beta$-catenin mRNA expression (Figure 4C,D). Moreover, Wnt recombinant plasmid reversed the promotion of lidocaine for E-cadherin and the inhibition of lidocaine for vimentin mRNA expression (Figure 4E,F). These data indicated that Wnt overexpression reversed the carcinostatic effect of lidocaine.

\section{Wnt inbibitor XAV-939 enhances the carcinostatic effect of lidocaine}

XAV-939, a selective inhibitor for the $\mathrm{Wnt} / \beta$-catenin pathway, was used to investigate the role of $\mathrm{Wnt} / \beta$-catenin signaling in the carcinostatic effect of lidocaine. We found that XAV-939 could inhibit cell migration compared with the control group. Moreover, cell migration was remarkably reduced in XAV-939 + $100 \mu \mathrm{M}$ lidocaine-treated group compared with that in the $100 \mu \mathrm{M}$ lidocaine-treated group (Figure 5A). Transwell assay showed that XAV-939 could significantly inhibited the cell invasion capacity, while the cell invasion capacity in XAV-939 + $100 \mu \mathrm{M}$ lidocainetreated group was substantially suppressed compare with that in the $100 \mu \mathrm{M}$ lidocaine-treated group (Figure 5B). The qRT-PCR data demonstrated that XAV-939 could obviously downregulate the expression level of Wnt and $\beta$-catenin mRNA compared with the control group, while the expression level of $\mathrm{Wnt}$ and $\beta$-catenin mRNA in XAV$939+100 \mu M$ lidocaine-treated group, was considerably downregulated compared with that in the $100 \mu \mathrm{M}$ lidocaine-treated group (Figure 5C,D). In addition, XAV939 could increase the expression of E-cadherin mRNA. When ovarian cancer cells were treated by XAV-939 + 100 $\mu M$ lidocaine, the expression level of E-cadherin mRNA was remarkably upregulated compared with that in the $100 \mu \mathrm{M}$ lidocaine-treated group (Figure 5E). XAV-939 also decreased the expression level of vimentin mRNA. XAV-939 + $100 \mu M$ lidocaine-treated ovarian cancer cells significantly downregulated the expression level of vimentin mRNA compared with that in the $100 \mu \mathrm{M}$ lidocaine-treated group (Figure $5 F$ ). These data indicated that $\mathrm{Wnt} / \beta$-catenin inhibitor XAV-939 could enhance the carcinostatic effect of lidocaine.

\section{Discussion}

The present study demonstrated that lidocaine could regulate the progression of ovarian cancer through inhibiting proliferation, migration, and invasion and inducing apoptosis of ovarian cancer cells in a dosedependent manner. $\mathrm{Wnt} / \beta$-catenin signaling was involved in the suppression of EMT progression of ovarian cancer cells, which resulted in the downregulation of Snail and vimentin, as well as the upregulation of E-cadherin. Furthermore, overexpressed Wnt could reverse the carcinostatic effect of lidocaine, while Wnt inhibitor XAV-939 enhanced the carcinostatic effect of lidocaine. These data indicated that lidocaine could inhibit proliferation and metastasis EOC by the $\mathrm{Wnt} / \beta$-catenin pathway.

Lidocaine, as a local anesthetic, has been found to have anticancer actions in a wide range of cancer cells. It does not only inhibit tumor cell growth in colon adenocarcinoma, bladder cancer, and gastric cancer, but also represses 
A

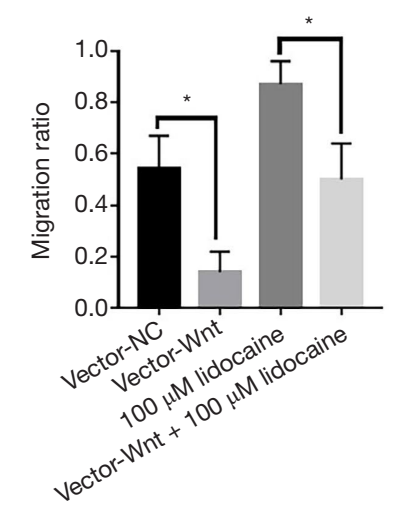

C

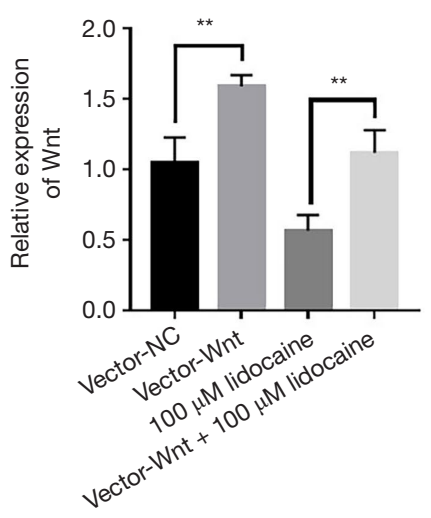

E

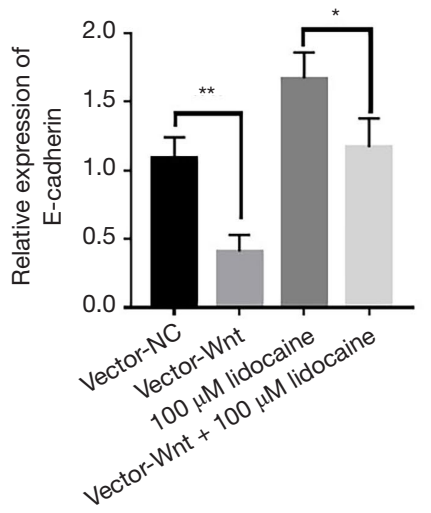

B
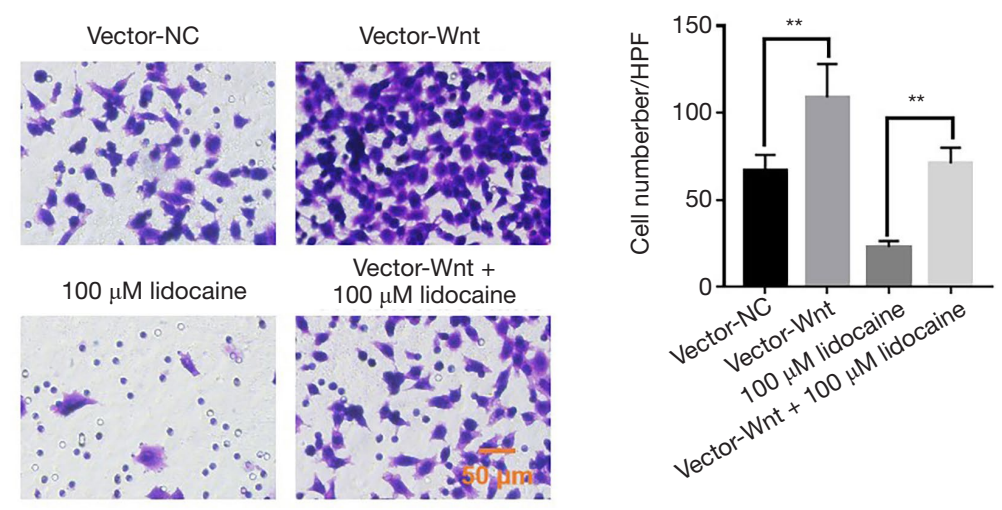

D

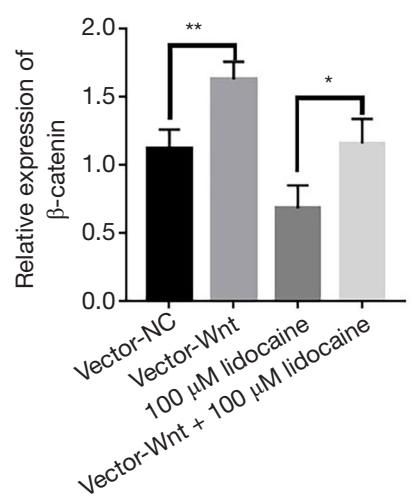

$\mathrm{F}$

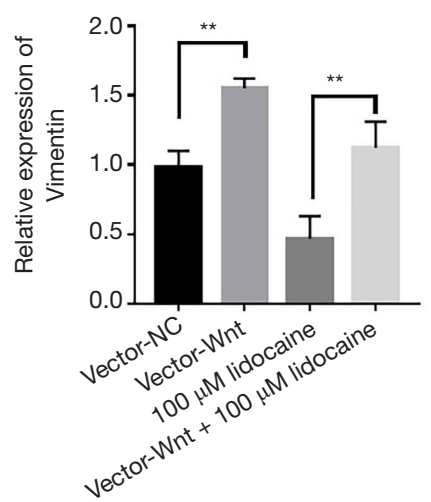

Figure 4 Overexpression of Wnt reduced the antitumor effect of lidocaine. (A) Migration distance was measured in Wnt recombinant plasmid, blank plasmid, $100 \mu \mathrm{M}$ lidocaine, and Wnt recombinant plasmid + $100 \mu \mathrm{M}$ lidocaine-treated group, by cell scratch test. (B) Cell invasion was detected in Wnt recombinant plasmid, blank plasmid, $100 \mu \mathrm{M}$ lidocaine, and Wnt recombinant plasmid + 100M lidocainetreated group by Transwell assay. (C,D,E,F) Expression of Wnt, $\beta$-catenin, E-cadherin, and vimentin was examined in Wnt recombinant plasmid, blank plasmid, $100 \mu \mathrm{M}$ lidocaine, and Wnt recombinant plasmid + $100 \mu \mathrm{M}$ lidocaine-treated group by quantitative reverse transcription polymerase chain reaction test. ${ }^{*} \mathrm{P}<0.05,{ }^{* *} \mathrm{P}<0.01$ : other groups compared with vector-NC group. 
A

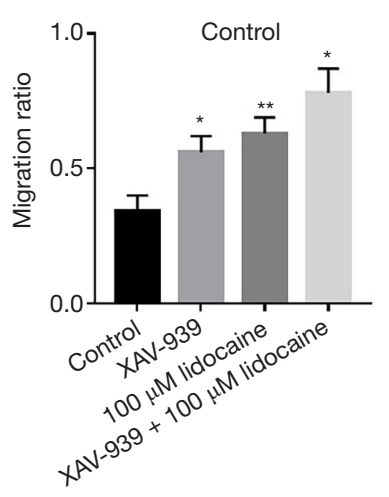

C

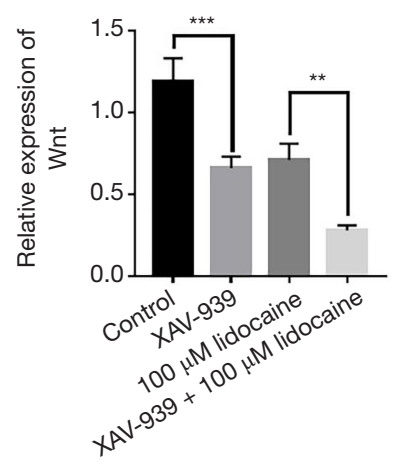

E

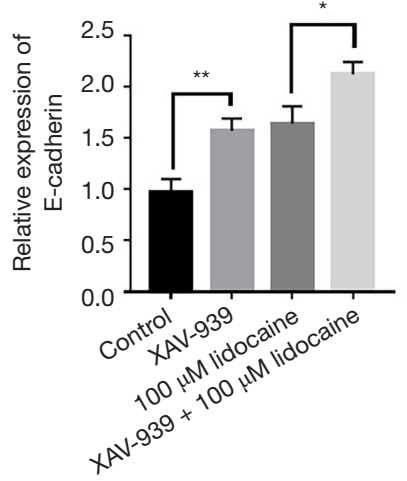

B

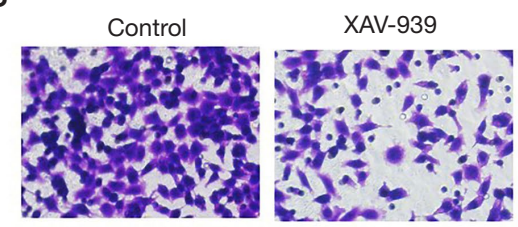

XAV-939+

$100 \mu \mathrm{M}$ lidocaine

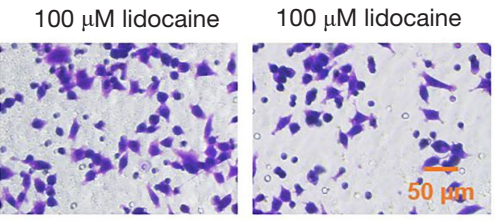

D

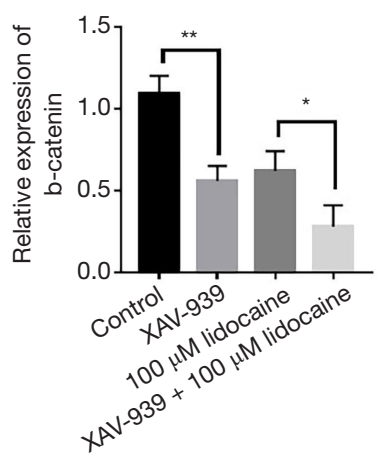

$\mathrm{F}$

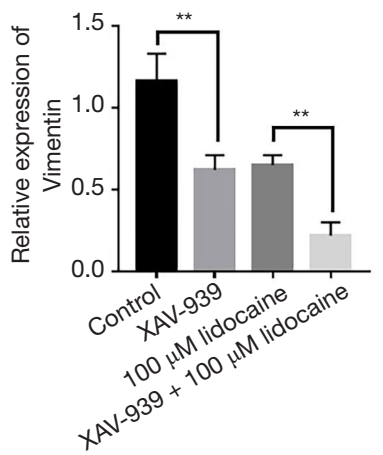

Figure 5 Wnt inhibitor XAV-939 enhanced the antitumor effect of lidocaine. (A) Migration distance was measured in PBS, XAV-939, 100 M lidocaine, and XAV-939 + $100 \mu M$ lidocaine-treated group by cell scratch test. (B) Cell invasion was detected in PBS, XAV-939, $100 \mu M$ lidocaine, and XAV-939 + $100 \mu \mathrm{M}$ lidocaine + treated group by Transwell assay. (C) Expression of Wnt was examined in PBS, XAV-939, 100 $\mu \mathrm{M}$ lidocaine, and XAV-939 + $100 \mu \mathrm{M}$ lidocaine-treated group by quantitative reverse transcription polymerase chain reaction (qRT-PCR) test. (D) Expression of $\beta$-catenin was examined in PBS, XAV-939, $100 \mu M$ lidocaine, and XAV-939 + $100 \mu M$ lidocaine-treated group by qRT-PCR test. (E) Expression of E-cadherin was examined in PBS, XAV-939, $100 \mu M$ lidocaine, and XAV-939 + $100 \mu M$ lidocaine-treated group by qRT-PCR test. (F) Expression of Wnt was examined in PBS, XAV-939, $100 \mu M$ lidocaine, and XAV-939 + $100 \mu M$ lidocainetreated group by qRT-PCR test. ${ }^{*} \mathrm{P}<0.05,{ }^{* *} \mathrm{P}<0.01,{ }^{* * *} \mathrm{P}<0.001$ : other groups compared with control group. PBS: phosphate buffer saline. 
tumor metastasis by inhibiting migration and invasion $(12-15)$. We also found that lidocaine inhibits proliferation, migration, and invasion, and induces apoptosis in ovarian cancer cells in a dose-dependent manner. In addition, Snail, E-cadherin, and vimentin, markers of the EMT process, were more significantly regulated as the lidocaine concentration increased compared with the control group, and EMT-associated genes matrix metalloprotein (MMP)7 and matrix metalloprotein (MMP)-9 were downregulated by lidocaine. These findings in EOC are similar to those of lung cancer, in which lidocaine was found to upregulate the cleavage of pro-apoptotic protein caspase-3, as well as altering EMT-related proteins MMP-9 and vimentin (23). Further, lidocaine is safe drug with little side effects. In one study of intravenous lidocaine for cancer pain, the most common side effects of lidocaine were drowsiness, perioral numbness, nausea, and slight fluctuations in blood pressure. It is safe and effective in cancer patients (24).

Wnt signaling is one of the key cascades regulating carcinogenesis $(21,25)$. The canonical Wnt pathway is commonly $\beta$-catenin-dependent signaling $(26,27)$. We found that lidocaine dose dependently inhibits the expression level of Wnt mRNA in ovarian cancer cells. $\beta$-Catenin is a crucial signaling transducer in Wnt signaling $(28,29)$; therefore, we further examined the expression level of $\beta$-catenin mRNA by qRT-PCR and found that $\beta$-catenin mRNA also had a low expression in ovarian cancer cells in the lidocainetreated group compared with the control group. Moreover, overexpressed Wnt reduced the antitumor effect of lidocaine by Wnt recombinant plasmid. In human cancers, $\mathrm{Wnt} / \beta$-catenin signaling is highly activated, which has led to the development of various Wnt signaling inhibitors for cancer therapies (30). We also found that Wnt inhibitor XAV-939 could enhance the antitumor effect of lidocaine, which decreased EMT progression and ovarian cancer. We demonstrated that lidocaine could inhibit the proliferation and metastasis of EOC through the $\mathrm{Wnt} / \beta$-catenin pathway. Further, lidocaine has been reported to promote ovarian and breast cancer cell ferroptosis via miR-382-5p/SLC7A11 axis (31). Lidocaine could promote thyroid cancer cells apoptosis via MAPK pathway (32). The invasion and migration of breast cancer, prostatic cancer and ovarian cancer cells with TRPV6-expressing could be inhibited by lidocaine, while this inhibition was associated with calcium influx reduction (12). In addition, lidocaine was also found to significantly inhibit $\mathrm{C}-\mathrm{X}$-C motif chemokine receptor 4 (CXCR4) signaling, EGFR signaling, and the MAP kinase kinase 1 (MEK)/extracellular regulated protein kinases
(ERK) and nuclear factor kappa-B (NF-кB) pathways, as well as activate the phosphatidylinositol 3-kinase (PI3K)/ Protein Kinase B (Akt) pathway $(6,15,16,23)$. Thus, lidocaine could exert anti-EOC effect via multi signal pathways.

\section{Conclusions}

We demonstrated that lidocaine could inhibit proliferation, migration, and invasion, and induce apoptosis in ovarian cancer cells in a dose-dependent manner to regulate the progression of ovarian cancer. It also could suppress the EMT progression of ovarian cancer through the Wnt/ $\beta$-catenin pathway. Wnt inhibitor XAV-939 further enhanced the antitumor effect of lidocaine.

\section{Acknowledgments}

Funding: None.

\section{Footnote}

Reporting Checklist: The authors have completed the MDAR reporting checklist. Available at https://dx.doi. org/10.21037/tcr-21-1047

Data Sharing Statement: Available at https://dx.doi. org/10.21037/tcr-21-1047

Conflicts of Interest: All authors have completed the ICMJE uniform disclosure form (available at https://dx.doi. org/10.21037/tcr-21-1047). The authors have no conflicts of interest to declare.

Ethical Statement: The authors are accountable for all aspects of the work in ensuring that questions related to the accuracy or integrity of any part of the work are appropriately investigated and resolved. The study was conducted in accordance with the Declaration of Helsinki (as revised in 2013). Institutional ethical approval and informed consent were waived.

Open Access Statement: This is an Open Access article distributed in accordance with the Creative Commons Attribution-NonCommercial-NoDerivs 4.0 International License (CC BY-NC-ND 4.0), which permits the noncommercial replication and distribution of the article with the strict proviso that no changes or edits are made and the 
original work is properly cited (including links to both the formal publication through the relevant DOI and the license). See: https://creativecommons.org/licenses/by-nc-nd/4.0/.

\section{References}

1. Lheureux S, Gourley C, Vergote I, et al. Epithelial ovarian cancer. Lancet 2019;393:1240-53.

2. Webb PM, Jordan SJ. Epidemiology of epithelial ovarian cancer. Best Pract Res Clin Obstet Gynaecol 2017;41:3-14.

3. Ferlay J, Soerjomataram I, Dikshit R, et al. Cancer incidence and mortality worldwide: sources, methods and major patterns in GLOBOCAN 2012. Int J Cancer 2015;136:E359-86.

4. Lheureux S, Braunstein M, Oza AM. Epithelial ovarian cancer: Evolution of management in the era of precision medicine. CA Cancer J Clin 2019;69:280-304.

5. Peres LC, Cushing-Haugen KL, Köbel M, et al. Invasive Epithelial Ovarian Cancer Survival by Histotype and Disease Stage. J Natl Cancer Inst 2019;111:60-8.

6. Jiang R, Liao J, Yang MC, et al. Lidocaine mediates the progression of cerebral ischemia/reperfusion injury in rats via inhibiting the activation of NF- $\mathrm{kB}$ p 65 and $\mathrm{p} 38$ MAPK. Ann Transl Med 2020;8:548.

7. Cata JP, Ramirez MF, Velasquez JF, et al. Lidocaine Stimulates the Function of Natural Killer Cells in Different Experimental Settings. Anticancer Res 2017;37:4727-32.

8. Yang X, Yang LX, Wu J, et al. Treatment of lidocaine on subacute thyroiditis via restraining inflammatory factor expression and inhibiting pyroptosis pathway. J Cell Biochem 2019. [Epub ahead of print]. doi: 10.1002/ jcb.27675.

9. Sisti MS, Nishida F, Zanuzzi CN, et al. Lidocaine protects neurons of the spinal cord in an excitotoxicity model. Neurosci Lett 2019;698:105-12.

10. Li J, Zhu X, Yang S, et al. Lidocaine Attenuates Cognitive Impairment After Isoflurane Anesthesia by Reducing Mitochondrial Damage. Neurochem Res 2019;44:1703-14.

11. Leng $T$, Lin $S$, Xiong $Z$, et al. Lidocaine suppresses glioma cell proliferation by inhibiting TRPM7 channels. Int J Physiol Pathophysiol Pharmacol 2017;9:8-15.

12. Jiang $\mathrm{Y}$, Gou $\mathrm{H}, \mathrm{Zhu} J$, et al. Lidocaine inhibits the invasion and migration of TRPV6-expressing cancer cells by TRPV6 downregulation. Oncol Lett 2016;12:1164-70.

13. Tat T, Jurj A, Selicean C, et al. Antiproliferative effects of propofol and lidocaine on the colon adenocarcinoma microenvironment. J BUON 2019;24:106-15.

14. Yang X, Zhao L, Li M, et al. Lidocaine enhances the effects of chemotherapeutic drugs against bladder cancer. Sci Rep 2018;8:598.

15. Sui $\mathrm{H}$, Lou A, Li Z, et al. Lidocaine inhibits growth, migration and invasion of gastric carcinoma cells by upregulation of miR-145. BMC Cancer 2019;19:233.

16. D'Agostino G, Saporito A, Cecchinato V, et al. Lidocaine inhibits cytoskeletal remodelling and human breast cancer cell migration. Br J Anaesth 2018;121:962-8.

17. Yang $\mathrm{Q}, \mathrm{Zhang} \mathrm{Z}, \mathrm{Xu} \mathrm{H}$, et al. Lidocaine alleviates cytotoxicity-resistance in lung cancer A549/DDP cells via down-regulation of miR-21. Mol Cell Biochem 2019;456:63-72.

18. Zhang L, Hu R, Cheng Y, et al. Lidocaine inhibits the proliferation of lung cancer by regulating the expression of GOLT1A. Cell Prolif 2017. doi: 10.1111/cpr.12364.

19. Liu C, Yu M, Li Y, et al. Lidocaine inhibits the metastatic potential of ovarian cancer by blocking $\mathrm{NaV} 1.5$-mediated EMT and FAK/Paxillin signaling pathway. Cancer Med 2021;10:337-49.

20. Li J, Zhao R, Guo Y, Fang R. EMG1 interacts with NOP14 to regulate the growth, migration, and invasion of melanoma cells via the Wnt/ -catenin pathway. Transl Cancer Res 2020;9:3669-79.

21. Zhan T, Rindtorff N, Boutros M. Wnt signaling in cancer. Oncogene 2017;36:1461-73.

22. Dodge ME, Moon J, Tuladhar R, et al. Diverse chemical scaffolds support direct inhibition of the membranebound O-acyltransferase porcupine. J Biol Chem 2012;287:23246-54.

23. Sun H, Sun Y. Lidocaine inhibits proliferation and metastasis of lung cancer cell via regulation of miR539/EGFR axis. Artif Cells Nanomed Biotechnol 2019;47:2866-74.

24. Peixoto RD, Hawley P. Intravenous lidocaine for cancer pain without electrocardiographic monitoring: a retrospective review. J Palliat Med 2015;18:373-7.

25. Haseeb M, Pirzada RH, Ain QU, et al. Wnt Signaling in the Regulation of Immune Cell and Cancer Therapeutics. Cells 2019;8:1380.

26. Qu Y, Kalland KH, Ke X. Small molecule induces Wnt asymmetry in cancer. Cell Cycle 2017;16:141-2.

27. Dey N, Barwick BG, Moreno CS, et al. Wnt signaling in triple negative breast cancer is associated with metastasis. BMC Cancer 2013;13:537.

28. Li VS, Ng SS, Boersema PJ, et al. Wnt signaling through inhibition of $\beta$-catenin degradation in an intact Axin1 
complex. Cell 2012;149:1245-56.

29. MacDonald BT, Tamai K, He X. Wnt/beta-catenin signaling: components, mechanisms, and diseases. Dev Cell 2009; 17:9-26.

30. Jung YS, Park JI. Wnt signaling in cancer: therapeutic targeting of Wnt signaling beyond $\beta$-catenin and the destruction complex. Exp Mol Med 2020;52:183-91.

31. Sun D, Li YC, Zhang XY. Lidocaine Promoted Ferroptosis by Targeting miR-382-5p /SLC7A11 Axis in Ovarian and Breast Cancer. Front Pharmacol 2021;12:681223.

32. Chang YC, Hsu YC, Liu CL, et al. Local anesthetics induce apoptosis in human thyroid cancer cells through the mitogen-activated protein kinase pathway. PLoS One 2014;9:e89563.

(English Language Editor: R. Scott)

Cite this article as: Sun M, Huang S, Gao Y. Lidocaine inhibits the proliferation and metastasis of epithelial ovarian cancer through the Wnt/ $\beta$-catenin pathway. Transl Cancer Res 2021;10(7):3479-3490. doi: 10.21037/tcr-21-1047 